\title{
Potentially Programmable Virtual Phase Plate for Electron Beams
}

\author{
Maurice Krielaart $^{1 *}$ and Pieter Kruit ${ }^{1}$ \\ 1. Delft University of Technology, Department of Imaging Physics, Delft, The Netherlands \\ * Corresponding author: m.a.r.krielaart@tudelft.nl
}

In light optics, the introduction of the spatial light modulator (SLM) enabled new image modalities, because of the added flexibility in (dynamically) shaping the optical wave front [1]. Unfortunately, such a versatile wave front shaper is not yet realized for application inside an electron microscope. Despite the many successes of utilizing static phase plates in the electron microscope, dynamic control over the full wave front by development of a programmable transmission phase plate is still in an early stage [2]. Some of the technological barriers are charging, contamination and optical transmissivity.

We present here an approach complementary to the above, were instead of manipulating the local phase by transmission through a patterned potential, we reflect the electron wave front on a topological- or charge-patterned mirror. Just in front of the mirror structure the effect of the mirror topology or charge distribution results in a spatial modulation of the electric field. The latter induces a path length difference within the reflected electron wave front and thus the ability to imprint a phase.

The use of mirrors in a commercial electron microscope requires to take the beam off-axis, perform the manipulation and place the beam back onto the optical axis. For instance, in LEEM a 90 degree-beam separator is used for this purpose [3]. An alternative method is recently suggested that relies on microfabricated electron optical devices, the use of which reduces the deflection angle of the beam separator below $100 \mathrm{mrad}$ [4]. The latter is used as a basis of the following.

We present a method here, in which the beam is taken away from the optical axis and later inserted again, with the desired phase manipulation, at exactly the same position. Hence, we speak of a virtual phase plate. The electron trajectories are shown schematically in Figure 1(a). Initially, the beam is focused onto a common cross-over plane. The beam is then electrostatically deflected towards the 'mirror axis', that runs parallel to, but is optically shielded from, the optical axis. A beam separator with crossed electrostatic and magnetic fields is used for alignment of the beam with the mirror axis. The beam is then spread out over and reflected by a mirror and focused onto the common cross-over plane of the mirror axis. The process repeats in reverse order at the mirror positioned at the top. This mirror carries the topological or charge-pattern. Finally, the now twice mirrored beam is deflected back onto the optical axis, where it has now acquired the desired phase pattern.

In order to design a mirror pattern that yields the desired effect, we are currently designing a numerical method. This method relies on application of Fresnel propagation of the desired amplitude and phase at the image plane back towards the mirror plane. The obtained wave front is next spectrally analysed and for every wave number the matching pattern amplitude is calculated. For this final step, the WKB method is used to couple the electric field in front of the mirror to the phase change that result in the electron wave front because of it [5].

In Figure 1(b-e) outputs of various steps of the numerical method are shown. In this example, we seek for a pattern that would result in an image plane with electron vortex beams of order $+/-1$. First, the use 
of the Fresnel propagator is validated by comparison of the obtained wave front at the mirror plane with existing holograms for generation of the aforementioned vortex beam [6]. The next obtained pattern topology is shown but it should be noted that this is an early preliminary result at this point.

The realization of a virtual phase plate faces technological challenges that include the fabrication of the pattern topology, electron beam separators and optical shielding of two nearby parallel axis. The successful application of the presented design inside a transmission electron microscope faces the additional challenges related to reflecting an electron beam with energies in excess of $100 \mathrm{keV}$. For this reason, initial experiments will focus on experiments at low beam energies of only a few keV.

Finally, we notice that the bottom mirror offers a degree of freedom that is not yet discussed. This second mirror may allow for full manipulation of both phase and amplitude of the beam.

\section{References:}

[1] C. Maurer, A Jesacher, S. Bernet and M. Ritsch-Marte, Laser Photonics Rev. 5 (2011), p. 81-101.

[2] J. Verbeeck et al, Ultramicroscopy 190 (2018), p. 58-65.

[3] R. M. Tromp et al, Ultramicroscopy 110 (2010), p. 852-861.

[4] H. Dohi and P. Kruit, Ultramicroscopy 189 (2018), p. 1-23.

[5] M.A.R. Krielaart and P. Kruit, Physical Review A 98 (2018), p. 063806.

[6] J. Verbeeck, H. Tian and P. Schattschneider, Nature 467 (2010), p. 301-304.

[7] The authors acknowledge funding from the Netherlands Organisation for Scientific Research.

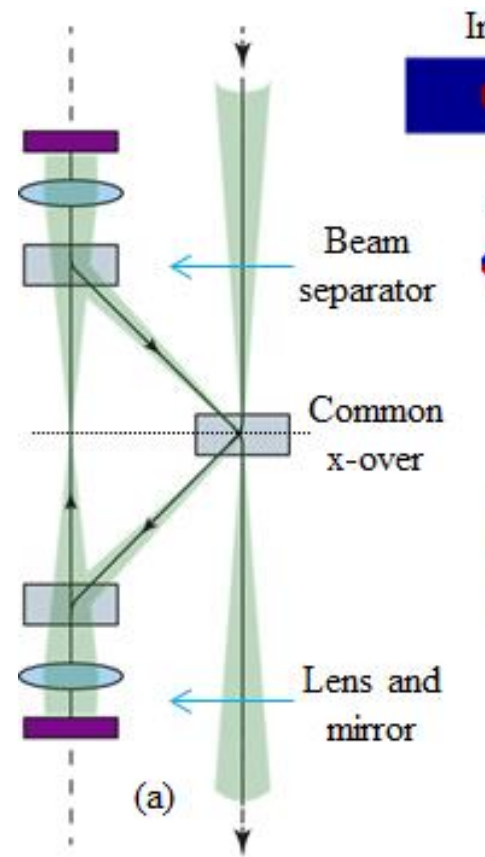

Image amplitude distribution

Imase distribution

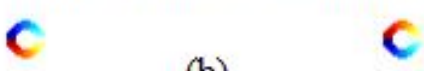

(b)

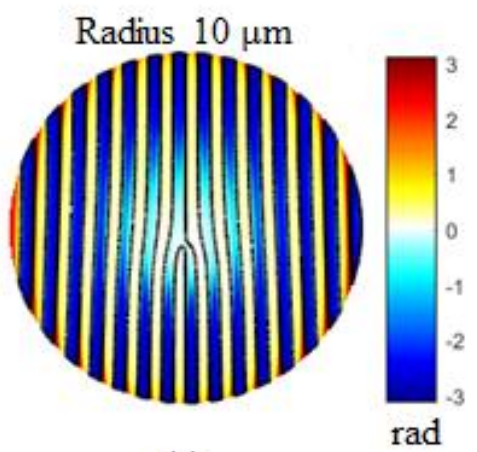

(c)

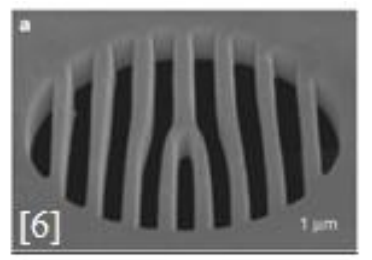

(d)

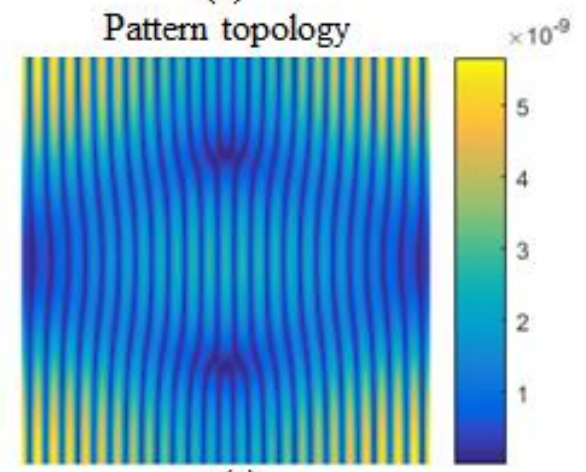

(e)

Figure 1. (a) Schematic electron beam trajectory through the virtual phase plate setup, with labels indicating the various electron optical components (see text). (b) Amplitude and phase distribution of a vortex beam with unity orbital angular momentum (OAM) at the image plane. (c) Wave front phase angles (colour map) and zero-phase angle contour for comparison to (d) existing holograms to produce said beam [6]. (e) Preliminary result of a calculated mirror topology for a beam energy of $2 \mathrm{keV}$. 